\title{
Model-Based Estimation of Cardiac Output and Total Peripheral Resistance
}

\author{
TA Parlikar ${ }^{1}$, T Heldt $^{1}$, GV Ranade ${ }^{1,2}$, GC Verghese ${ }^{1}$ \\ ${ }^{1}$ Massachusetts Institute of Technology, Cambridge, MA, USA \\ ${ }^{2}$ University of California, Berkeley, CA, USA
}

\begin{abstract}
We describe a novel model-based approach to estimate cardiac output $(\mathrm{CO})$ and total peripheral resistance $(T P R)$ continuously from peripheral arterial blood pressure $(A B P)$ waveforms. Our method exploits the intra-beat and inter-beat variability in $A B P$ to estimate the lumped time constant of a beat-to-beat averaged Windkessel model of the arterial tree, from which we obtain an uncalibrated estimate of $\mathrm{CO}$. To estimate absolute $\mathrm{CO}$, we determine the lumped arterial compliance using calibration data, and assuming either constant or state-dependent compliance. We applied our method to a porcine data set in which stroke volume was measured with an ultrasonic flowmeter. We obtain root-mean-square normalized errors of 11-13\% across all pigs, lower than those obtained on the same data set using various other estimation methods. The CO estimates, and TPR estimates derived from them track intravenous drug infusions quite closely.
\end{abstract}

\section{Introduction}

Cardiac output (CO) is a key hemodynamic variable that is commonly used to establish differential diagnoses, monitor disease progression, and titrate therapy in many cardiovascular conditions. The current clinical gold-standard for measuring $\mathrm{CO}$ is intermittent thermodilution, a highly invasive procedure in which a catheter is advanced to the pulmonary artery, a bolus of cold saline is injected into the circulation, and the blood's temperature profile is observed as a function of time. Due to its high degree of invasiveness, this procedure is usually reserved for only the sickest of patients, and even in critical care its benefit is increasingly questioned [1]. Rather than intermittently measuring average cardiac output invasively via thermodilution, many attempts have been made to estimate $\mathrm{CO}$ from the arterial blood pressure (ABP) waveform [2, 3, 4, 5, 6, 7], using models of the arterial system, one of the most basic of which is the Windkessel model of the arterial tree [2] (see Figure 1 and Section 2).

An entire class of algorithms is based on analyzing the pressure pulse morphology in the context of Windkessel-

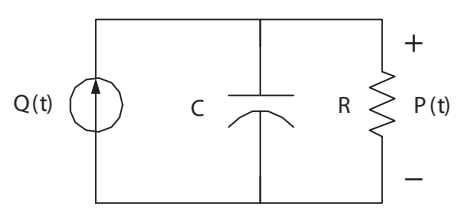

Figure 1. Circuit representation for the Windkessel model.

like models [3, 4, 5, 8]. Each of these algorithms assumes that the arterial tree behaves like a Windkessel on an intracycle timescale; $\mathrm{CO}$ is estimated using morphological features of each individual ABP wavelet (such as systolic, mean, and diastolic ABP). More recently, Mukkamala and co-workers [7, 9] estimated relative changes in cardiac output from the inter-cycle (or beat-to-beat) variations of the ABP waveform, using these to determine the impulse response function of the Windkessel model and, from it, the time constant of arterial outflow. Knowing the latter, the authors determined proportional $\mathrm{CO}$, from which absolute $\mathrm{CO}$ can be obtained via calibration with a single or multiple reference $\mathrm{CO}$ measurements.

Our own interest in estimating $\mathrm{CO}$ and TPR derives from our work in the area of cycle-averaged models of the cardiovascular system $[10,11]$, where again the focus is on inter-cycle variation. By applying a cycle-averaging operation to the Windkessel model, we will show how to arrive at an estimation scheme that uses both intra- and inter-cycle information to determine $\mathrm{CO}$.

\section{Methods}

\section{Windkessel model}

The Windkessel model describes the basic morphology of an arterial pressure pulse [2]. It lumps the distributed resistive and capacitive properties of the entire arterial tree into two elements, as seen in the electrical circuit analog in Figure 1: a single resistor $R$, representing total peripheral resistance (TPR), and a single capacitor $C$, representing the aggregate elastic properties of all systemic arteries.

The differential equation representing the Windkessel 
circuit at time $t$ is given by

$$
C \frac{d P(t)}{d t}+\frac{P(t)}{R}=Q(t)
$$

where $P(t)$ represents arterial blood pressure at the aortic root at time $t$. This equation shows that the time constant $\tau=R C$ governs the intra-cycle dynamics of the Windkessel model. The same time constant also governs the inter-cycle dynamics, as noted in [7, 10, 11].

The pumping action of the heart is represented by an impulsive current source $Q(t)$ that deposits a stroke volume $\mathrm{SV}_{n}$ into the arterial system during the $n^{\text {th }}$ cardiac cycle:

$$
Q(t)=\sum_{n} \mathrm{SV}_{n} \cdot \delta\left(t-t_{n}\right)
$$

where $t_{n}$ is the onset time of the $n^{\text {th }}$ beat and $\delta(t)$ is the unit Dirac impulse. It then follows by integrating (1) over just the (infinitesimal) ejection phase that

$$
\mathrm{SV}_{n}=C \cdot \mathrm{PP}_{n}
$$

where $\mathrm{PP}_{n}$ is the pulse pressure in the $n^{\text {th }}$ cardiac cycle, given by $\mathrm{PP}_{n}=\mathrm{SAP}_{n}-\mathrm{DAP}_{n}$, with $\mathrm{SAP}_{n}$ and $\mathrm{DAP}_{n}$ being respectively systolic and diastolic arterial pressure in that cycle.

We define $T_{n}$ to be the duration of the $n^{\text {th }}$ cardiac cycle, i.e., the beat that begins at time $t_{n}$ and ends at time $t_{n+1}$ (so $T_{n}=t_{n+1}-t_{n}$ ). It follows that the average cardiac output in the $n^{\text {th }}$ cycle is given by

$$
\mathrm{CO}_{n}=\frac{\mathrm{SV}_{n}}{T_{n}}=C_{n} \frac{\mathrm{PP}_{n}}{T_{n}}
$$

where the first equality is simply the definition and the second follows on substituting from (3).

\section{Beat-to-beat averaged model}

Given pulse pressure, (4) can be used to estimate values of cardiac output. However, since the relation (3) is based entirely on the essentially instantaneous ejection period assumed in this model, the $\mathrm{CO}$ estimate obtained via (4) does not take advantage of information from the remainder of the cardiac cycle that could be harnessed to provide a better-conditioned estimate. Specifically, the fact that (1) interrelates the variables during the entire cardiac cycle, and indeed from one cycle to the next, has not been exploited in the development so far.

To better reflect intra-cycle and inter-cycle behavior, we average (1) over the entire cardiac cycle rather than just the ejection phase. Considering $R$ and $C$ to be constant within each cardiac cycle, but allowing them to vary from cycle to cycle, this averaging yields the following relation over the $n^{\text {th }}$ cycle [11]:

$$
C_{n} \frac{\Delta P_{n}}{T_{n}}+\frac{\bar{P}_{n}}{R_{n}}=\mathrm{CO}_{n}
$$

where

$$
\Delta P_{n}=P\left(t_{n+1}\right)-P\left(t_{n}\right)
$$

is the the beat-to-beat pressure change at the onset times, and

$$
\bar{P}_{n}=\frac{1}{T_{n}} \int_{t_{n}}^{t_{n+1}} P(t) d t
$$

is the average ABP over the $n^{\text {th }}$ cycle. Note that (5) is a natural discrete-time counterpart to (1), with the first and second terms now representing average flow through the capacitor and resistor, respectively, in the $n$-th cycle.

Combining (4) and (5), we obtain

$$
\frac{\Delta P_{n}}{T_{n}}+\frac{\bar{P}_{n}}{\tau_{n}}=\frac{\mathrm{PP}_{n}}{T_{n}},
$$

where $\tau_{n}=R_{n} C_{n}$ is the only unknown.

Because determination of central $\mathrm{PP}_{n}$ from peripheral pressure waveforms is problematic due to wave reflections, we use an expression presented in [6] to estimate $\mathrm{PP}_{n}$ in terms of the mean pressure $\overline{\mathrm{P}}_{n}$ in the $n$-th cycle and $\mathrm{DAP}_{n}$ :

$$
\mathrm{PP}_{n}=\alpha\left(\bar{P}_{n}-\mathrm{DAP}_{n}\right)
$$

where $\alpha$ is a constant. Assuming a triangular pulse shape yields $\alpha=2$, but our results for the $\mathrm{CO}$ estimate are not overly sensitive to the precise value of $\alpha$.

\section{Estimation}

Equation (8) can be used to estimate $1 / \tau_{n}$ from knowledge of the remaining quantities. Specifically, using a data window comprising an odd number of beats centered at $n$, and assuming $\tau_{n}$ to be essentially constant over this window, we invoke (8) for each of the beats in the window to obtain a set of linear equations in the single unknown $1 / \tau_{n}$. The least-square-error solution of this set yields the desired estimate. Repeating the process on a sliding window produces an estimate of $1 / \tau_{n}$ for every beat.

Cardiac output can now be estimated from (5), rewritten below to show dependence on $1 / \tau_{n}$ :

$$
\mathrm{CO}_{n}=C_{n}\left(\frac{\Delta P_{n}}{T_{n}}+\frac{\bar{P}_{n}}{\tau_{n}}\right)
$$

The conventional expression for $\mathrm{CO}_{n}$ neglects beat-to-beat variability and therefore omits the term $\Delta P_{n} / T_{n}$; it is thus actually valid only in cyclic steady state, while (10) holds more generally. The determination of $C_{n}$ using calibration information is discussed in the next subsection.

Total peripheral resistance is also an important cardiovascular variable. In the clinical setting, TPR is defined as the ratio of mean arterial blood pressure to cardiac output. However, taking into account beat-to-beat variability 
as in (10) and thereby accounting for transient flow into the arterial compliance, yields the modified expression

$$
R_{n}=\frac{\bar{P}_{n}}{\mathrm{CO}_{n}-C_{n} \frac{\Delta P_{n}}{T_{n}}} .
$$

\section{Calibration}

To compute $\mathrm{CO}_{n}$ using (10) we first need to estimate the compliance $C_{n}$ by using it to calibrate $\mathrm{CO}$ against one or more true or reference $\mathrm{CO}$ measurements. A natural calibration criterion is the root-mean-square-normalized error (RMSNE) at the calibration points. If the compliance $C_{n}$ is assumed to be a constant $C$, then it is straightforward to choose the $C$ that minimizes the RMSNE [11]. Mukkamala [7] instead used a mean calibration, dividing the mean of the true $\mathrm{CO}$ values by the mean of the estimated $\mathrm{CO}$ values at those points.

Considerably better results can be obtained by using a state-dependent model for $C_{n}$. A simple choice is to assume an affine dependence of $C_{n}$ on $\bar{P}_{n}$ :

$$
C_{n}=\gamma_{1}+\gamma_{2} \bar{P}_{n}
$$

The calibration can be performed using a least-squareerror solution to a linear system of equations [11]. Other parameterizations may also be used.

\section{Dataset}

We tested the algorithm outlined above on the porcine dataset used by Mukkamala [7]. The data contains measurements of ECG, radial arterial blood pressure (rABP), femoral arterial blood pressure (fABP), and aortic flow (AF), all sampled at $250 \mathrm{~Hz}$ with 16-bit amplitude resolution. From these waveforms we derived mean, systolic, and diastolic arterial blood pressures. True $\mathrm{CO}$ was calculated by averaging the AF waveform over each beat.

\section{Results}

Unless noted otherwise, the results reported below were generated using a 100-point state-dependent calibration to obtain $C_{n}$ for each animal. This represents less than $1 \%$ of each animal's data record, though our results change minimally if as few as 10 or as many as 1,000 points are used to calibrate.

Table 1 summarizes the error obtained for each animal using either the rABP or fABP waveforms to estimate CO. Our results show mean RMSNEs of about $12 \%$, which is lower than the $15 \%$ reported in the literature as being acceptable for clinical purposes [9]. Figure 2 shows the true and estimated $\mathrm{CO}, \mathrm{HR}$, mean rABP, true and estimated TPR, and drug infusions for Animal 1. The spikes in the $\mathrm{HR}$ and $R$ waveforms are a result of not filtering
Table 1. CO RMSNEs for each animal using 360-beat data windows. Different window sizes yield similar errors.

\begin{tabular}{|c||c|c|c|}
\hline Animal & $\begin{array}{c}\text { Number of } \\
\text { comparisons }\end{array}$ & $\begin{array}{c}\text { RMSNE (\%) } \\
\text { using rABP }\end{array}$ & $\begin{array}{c}\text { RMSNE (\%) } \\
\text { using fABP }\end{array}$ \\
\hline 1 & 14604 & 15.4 & 10.2 \\
2 & 14404 & 10.6 & 9.4 \\
3 & 12088 & 9.7 & 8.8 \\
4 & 18155 & 11.1 & - \\
5 & 14113 & 8.4 & 12.6 \\
6 & 9370 & 15.8 & 19.5 \\
\hline \hline Aggregate & 82734 & 11.9 & 12.1 \\
\hline
\end{tabular}
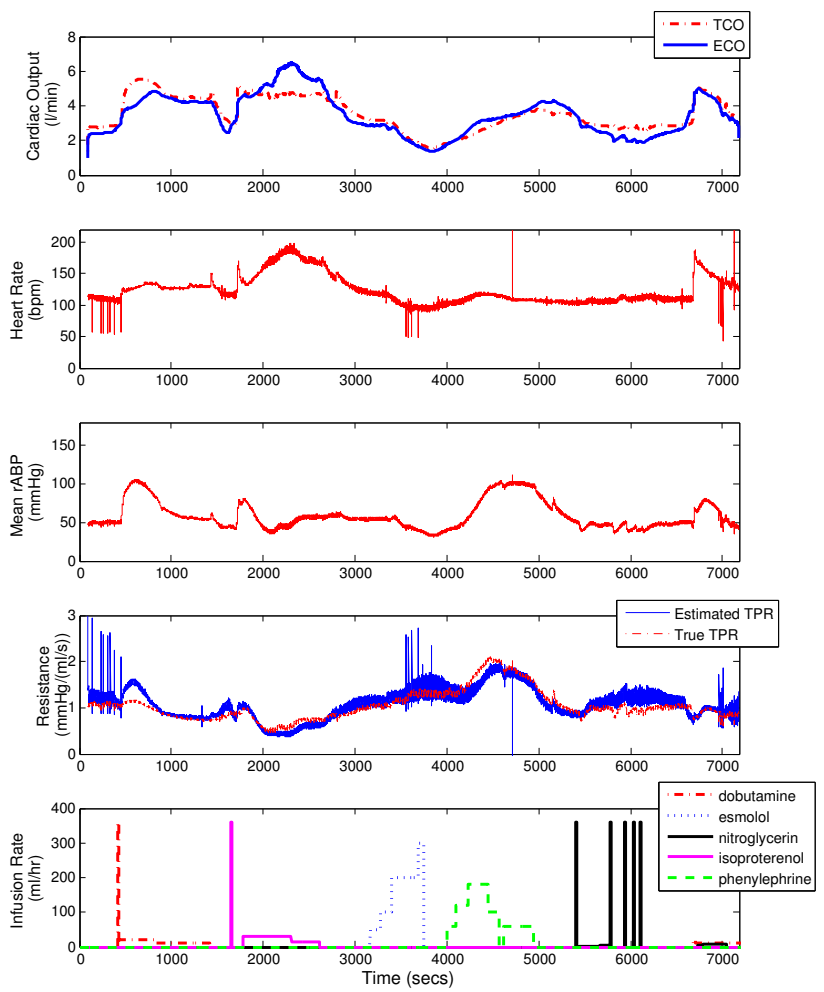

Figure 2. True (TCO) and estimated (ECO) cardiac output, HR, mean rABP, true and estimated TPR, and IV drugs for animal 1.

$T_{n}$. Estimated $\mathrm{CO}$ and TPR track true $\mathrm{CO}$ and TPR very well while all major hemodynamic variables are varied independently over a wide range. Furthermore, due to the continuous nature of our CO and TPR estimates, we track pharmacological interventions quite closely.

We compared our method to that of Mukkamala and several Windkessel-based CO estimation methods. To do a fair comparison, we used a mean calibration for all estimates. The results of this analysis are shown in Table 2. Overall, our method (even without the state-dependent calibration) and the methods proposed by Herd and Mukkamala produce essentially equivalent results, and they outperform the other methods tested. In data segments in which beat-to-beat variability is significant, as reflected by 
Table 2. Mean RMSNEs for various CO estimates reported in the literature.

\begin{tabular}{cccc}
\hline $\begin{array}{c}\text { CO Estimation } \\
\text { Method }\end{array}$ & $\mathrm{CO}_{n}=$ & $\begin{array}{c}\text { RMSNE (\%) } \\
\text { using rABP }\end{array}$ & $\begin{array}{c}\text { RMSNE (\%) } \\
\text { using fABP }\end{array}$ \\
\hline \hline Our Method & $(10)$ & 13.5 & 15.2 \\
Mukkamala [7] & $\mathrm{ARMA} \mathrm{model}_{n}$ & 14.0 & 15.0 \\
Herd [6] & $C \cdot \mathrm{HR}_{n} \cdot\left(\bar{P}_{n}-\mathrm{DAP}_{n}\right)$ & 14.0 & 15.9 \\
Modified Mean Pressure & $C \cdot \mathrm{HR}_{n} \cdot \bar{P}_{n}$ & 18.6 & 20.0 \\
Static Windkessel [8] & $C \cdot \mathrm{HR}_{n} \cdot\left(\mathrm{SAP}_{n}-\mathrm{DAP}_{n}\right)$ & 21.1 & 18.8 \\
Liljestrand and Zander [3] & $C \cdot \mathrm{HR}_{n} \cdot\left(\frac{\mathrm{SAP}_{n}-\mathrm{DAP}_{n}}{\mathrm{SAP}_{n}+\mathrm{DAP}_{n}}\right)$ & 30.0 & 25.1 \\
Mean Pressure & $\operatorname{scaled}_{n}$ & 31.6 & 33.6 \\
\hline
\end{tabular}

the ratio $\Delta P_{n} / \mathrm{PP}_{n}$, our method does substantially better than static pulse contour methods that solely analyze the intra-beat pulse shape [11].

\section{Conclusions}

In this paper, we described a novel model-based method for continuously estimating $\mathrm{CO}$ and TPR from peripheral arterial blood pressure waveforms. The method combines intra- and inter-beat information to obtain competitive estimates of CO and TPR. These estimates improve further on exploiting a state-dependent compliance model.

We applied our method, and several others reported in the literature, to porcine data in which reference CO measurements were available on a beat-by-beat basis. The aggregate estimation error of our $\mathrm{CO}$ estimates is among the lowest of all the methods tested. CO and TPR estimates tracked the animals' expected hemodynamic responses to intravenous drugs well.

We are currently refining our method and applying it to additional animal and human data sets.

\section{Acknowledgments}

This work was supported in part by the National Institute of Biomedical Imaging and Bioengineering of the National Institutes of Health through grant R01 EB 001659, and by Philips Research North America. We thank Prof. Ramakrishna Mukkamala of Michigan State University for making available the experimental data used in this study. We also thank Prof. Roger Mark, Prof. Richard Cohen, and Dr. Mohammed Saeed of the Harvard-MIT Division of Health Sciences and Technology for their helpful comments and encouragement over the course of this work.

\section{References}

[1] Rubenfeld G, McNamara-Aslin E, Rubinson L. The pulmonary artery catheter, 1967-2007: Rest in peace? JAMA 2007;298(4):458-461.
[2] Frank O. Die Theorie der Pulswelle. Z Biol 1927;85:91130.

[3] Liljestrand G, Zander E. Vergleichende Bestimmung des Minutenvolumens des Herzens beim Menschen mittels der Stickoxydulmethode und durch Blutdruckmessung. Z Exp Med 1928;59:105-122.

[4] Wesseling K, Jansen J, Settels J, Schreuder J. Computation of aortic flow from pressure in humans using a nonlinear, three-element model. J Appl Physiol 1993;74(5):25662573.

[5] Bourgeois M, Gilbert B, Donald D, Wood E. Characteristics of aortic diastolic pressure decay with application to continuous monitoring of changes in peripheral vascular resistance. Circ Res 1974;35(1):56-66.

[6] Herd J, Leclair N, Simon W. Arterial pressure pulse contours during hemorrhage in anesthetized dogs. J Appl Physiol 1966;21(6):1864-1868.

[7] Mukkamala R, Reisner A, Hojman H, Mark R, Cohen R. Continuous cardiac output monitoring by peripheral blood pressure waveform analysis. IEEE Trans Biomed Eng 2006; 53(3):459-467.

[8] Frank O. Schätzung des Schlagvolumens des menschlichen Herzenz auf Grund der Wellen- und Windkesseltheorie. Z Biol 1930;90:405-409.

[9] Lu Z, Mukkamala R. Continuous cardiac output monitoring in humans by invasive and noninvasive peripheral blood pressure waveform analysis. J Appl Physiol 2006; 101(2):598-608.

[10] Parlikar T, Heldt T, Verghese G. Cycle-averaged models of cardiovascular dynamics. IEEE Trans on Circ Syst I 2006; 53(11):2459-2468.

[11] Parlikar T. Modeling and Monitoring of Cardiovascular Dynamics for Patients in Critical Care. Ph.D. Thesis, Massachusetts Institute of Technology, Cambridge, MA, June 2007.

Address for correspondence:

George C. Verghese

MIT, Room 10-140K

77 Massachusetts Avenue

Cambridge, MA 02139, USA

verghese@mit.edu 